\title{
A Mixed Hybrid Algorithm for Integral Wind Farm Optimum Design
}

\author{
J. Castro Mora ${ }^{1}$, Á.G. González Rodríguez ${ }^{2}$, J.M. Riquelme Santos ${ }^{1}$, and M. Burgos Payán ${ }^{1}$ \\ ${ }^{1}$ Department of Electrical Engineering, University of Seville \\ Camino de los Descubrimientos, s/n - 41092 Sevilla, Spain \\ phone: +34954 487283 - fax: +34954 487284 - e-mail: jsantos@us.es, mburgos@us.es \\ ${ }^{2}$ Department of Electronic, University of Jaén \\ Escuela Politécnica Superior - Campus "Las Lagunillas" - 23071 Jaén, Spain \\ phone: +34 953212424 - fax: +34 953212400 - e-mail: agaspar@ujaen.es
}

\begin{abstract}
Nowadays, the weight of the generation of renewable energies has grown spectacularly with regard to other conventional energies. This is due to diverse factors: a bigger environmental concern (Kyoto protocol, white-book in $\mathrm{EU}$, etc.), the raising prices of the traditional fuels, etc. On the other hand, wind power has experienced a bigger grown, among the renewable energies. That is why the development of an effective tool for the design and lay-out of wind farms has a special relevance.
\end{abstract}

This paper present a mix of evolutionary algorithms to look for the optimum integral design of the wind park taking into account all the part involved: number of wind generators, the type and the height of the wind generators, its location, the number and location of substations, the best layout of lowvoltage and high-voltage lines among wind generators, substations and the existing transmission lines, etc.

Due to problem complexity, and as a first approach, the global optimization problem has been splitted in two main (uncoupled) parts, first the wind generators lay out and then the network configuration.

\section{Key words}

Wind farm, genetic algorithm, optimization techniques.

\section{Introduction}

The profitability of a wind farm installation investment depends on three main economical terms: initial investment, cost of operation and sale of the product (the generated electrical energy). In the case of a wind farm installation, these three economical investment components are difficult to evaluate, even simplifying the problem, because each term depends of a lot variables interrelated. Each type of wind generator has a different economic cost, but each one of them generates a different power. The individual wind turbine location affects the investment (foundations and interconnection), the interconnection, for example, affects the operation costs, and the wind generators lay-out to the potential electrical energy generation.

The objective of this communication presents the development of a new tool for the optimum design of wind farms. The optimization criterion, as in any economical investment, is the investment in the wind farm installation. The investment must be as profitable as possible.

Due to the complexity of the problem, and as a first approach, the global problem has been splitted in two main optimization sub-problems:

- $\quad$ Optimum wind generators lay out.

- Optimum wind farm network configuration.

From the economic point of view, the first problem, the wind generator sitting, is the most significant, as it is responsible of most part of the investment and it more affects to the production [1-3]. The second problem is similar to the design of a new radial network [4-7]. Both problems have been analyzed by means of economic functions that allow comparing the different terms related to the wind farm design and operation from the park.

After this introduction, the article has been structured in four parts. The following two sections introduce both sub-problems. A study case is analyzed in the following section and finally the main conclusions are summarized.

\section{A genetic-evolutive algorithm for wind turbine optimum sitting}

The objective function of the first problem is the maximization of the net present value (NPV). The NPV is used as an index to analyze and evaluate the profitability of the investment. This economic factor is usually used as an index that reveals if an investment is profitable or not.

The fitness function (NPV) can take a value positive, negative or null, corresponding to a profitable, not profitable or indifferent solution, respectively.

$N P V(x, i, t)=\frac{N_{1}(x)}{1+i}+\cdots+\frac{N_{t}(x)}{(1+i)^{t}}-I C(x)$

Where 
IC: Initial capital investment

$N_{k}$ : Net cash flow at year $k$

$i$ : Discount rate (capital cost)

$x$ : State vector with the location and height of wind generators

$t$ : Number of years spanned by the investment

The net cash flow at year $\left(N_{k}\right)$ is the value of the energy generated by all the wind generators in the year $k$.

The initial capital investment is worked out from the wind generators, the tower and the installation costs.

Therefore, the maximization of the NPV means the minimization of the investment and the maximization of the net cash flows (to maximize the generation of energy). Both terms depends on the number and type of wind generators and their tower height and position.

An evolutive algorithm is proposed to maximize the NPV function. Previously is necessary to calculate the energy generated in a year, taken into account the type and tower high of the generators, and the wind distribution at the wind farm allocation [8]. This problem is rather complex, non-linear and no derivable. So an evolutive algorithm seems to be very suitable to solve it.

Genetic algorithms are robust algorithms that find the minimum or the maximum of a function [9-10]. These algorithms use multiple directions of search instead of the only one direction, and use to work with a codified structure of the variables instead of his real values.

The genetic algorithms are basically a mechanism of search based on the species evolution. This evolution is based on the natural selection and survival of the individuals best adapted in relation to the fitness (objective) function. A genetic algorithm is an iterative process where the population is evolving and where all the individuals are possible solutions of the problem. The initial population can be settled heuristically or randomly. From this initial population the parents are selected. These parents, by means of crossings and mutation operations, give place to the following generation. The evolution used in this work is partially elitist because is always included the best individual of the previous population in the following generation. This way the population evolves towards the optimum solution of the problem.

An evolutive algorithm (EA) has been used to solve the placement and features of the wind generators. Five types of crossover operators have been developed: simple crossover, average crossover, union crossover, mixed union crossover and interchange crossover.

Once the crossovers have been done, the resulting solutions are mutated. When the population is confined in a local maximum, this operator leads to the creation of individuals out of this zone of local maximum looking for the global maximum. A regenerative algorithm is applied turning the unfeasible individual in feasible.
Genetic algorithms are robust optimum search techniques that find the minimum or the maximum of a function based on principles inspired from the natural genetic and evolution mechanisms observed in the nature. These algorithms use multiple paths of search instead of single point, using encoded solutions to the problem (variable values or genotypes), instead of their real values. Their main principle is the maintenance of a set of encoded solutions (population) that evolves in time, guiding the population towards the optimum solution [11]. They are based on the continuous Darwinian improvement cycle of evaluation, selection and reproduction of the best individuals. The initial population can be settled heuristically or randomly [10]. Genetic reproduction is performed by means of a few basic genetic operators, mainly crossover and mutation, that recombine highly fit individuals (best solutions). Evaluation is performed by means of a specific objective (fitness) function that depends on each particular optimization problem and is the objective function of the genetic algorithm. Individual selection is performed according to a selection strategy that chooses parents with probability proportional to their relative worth (fitness) and explores the solution space looking for better and better solutions.

As can be seen, genetic algorithms are, basically, mechanisms of search based on the species evolution. This evolution is based on the natural selection and survival of the best-adapted individuals, objectively evaluated with the fitness function.

\section{A. Codification}

An integer codification has been used to codify every possible solution of the problem. This integer codification has been made by means of a matrix formulation, where every column represents the characteristics of a wind generator, and every row represents: position of the wind generator in Cartesian coordinates $\left(X_{i}, Y_{i}\right)$, type of wind generator $\left(T_{i}\right)$ and tower height $\left(H_{i}\right)$ that constitute an individual solution.

The type of wind generator will be codified with a number, which will be the index in the generator database that uses the algorithm as an input. The above-mentioned database will content all the necessary information of the wind generators that can be installed in the wind farm (i.e. maximum and minimum height of the towers, capital cost, curve power-wind speed). Therefore, these matrixes have a variable number of columns, depending on the number of generators required by the codified individual solution, as shown in Fig. 1.

\begin{tabular}{ccccc} 
& \multicolumn{1}{c}{$\begin{array}{c}\text { 1st. } \\
\text { Generator }\end{array}$} & $\begin{array}{c}\text { 2nd. } \\
\text { Generator }\end{array}$ & \multicolumn{1}{c}{$\begin{array}{c}\text { k-th } \\
\text { Generator }\end{array}$} \\
\cline { 2 - 6 } x coordinate & $\boldsymbol{X}_{1}$ & $\boldsymbol{X}_{2}$ & $\ldots$ & $\boldsymbol{X}_{\boldsymbol{k}}$ \\
\cline { 2 - 5 } y coordinate & $\boldsymbol{Y}_{1}$ & $\boldsymbol{Y}_{2}$ & $\ldots$ & $\boldsymbol{Y}_{\boldsymbol{k}}$ \\
Generator type & $\boldsymbol{T}_{1}$ & $\boldsymbol{T}_{2}$ & $\ldots$ & $\boldsymbol{T}_{k}$ \\
\cline { 2 - 5 } Tower height & $\boldsymbol{H}_{1}$ & $\boldsymbol{H}_{2}$ & $\ldots$ & $\boldsymbol{H}_{k}$ \\
\cline { 2 - 6 }
\end{tabular}

Fig. 1. Codification of an individual. 
A family is represented by a set of individuals. Each individual in a family is codified as a $4 \mathrm{xkn}$ integer matrix (Fig 2.), being $k n$ the number of wind generators that constitute the individual solution (that could be variable $k 1, k 2 \ldots k n)$. Note that it is possible to find out individuals within a family with different number of wind generators, being necessary to assure this variability during the evolution of the EA. For this purpose, specific operators for crossing and mutation have been developed.

First Individual
\begin{tabular}{|c|c|c|c|}
\hline$X_{1}$ & $X_{2}$ & $\ldots$ & $X_{k 1}$ \\
\hline$Y_{1}$ & $Y_{2}$ & $\ldots$ & $Y_{k 1}$ \\
\hline$T_{1}$ & $T_{2}$ & $\ldots$ & $T_{k 1}$ \\
\hline$H_{1}$ & $H_{2}$ & $\ldots$ & $H_{k 1}$ \\
\hline$H_{1}$ & $H_{2}$ & $\ldots$ & $H_{k 2}$ \\
\hline$T_{1}$ & $Y_{2}$ & $\ldots$ & $Y_{k 2}$ \\
\hline$X_{1}$ & $X_{2}$ & $\ldots$ & $X_{k 2}$ \\
\hline$H_{1}$ & $H_{2}$ & $\ldots$ & $H_{k n}$ \\
\hline
\end{tabular}

Fig. 2. Codification of a family.

\section{B. Crossover operators}

The crossover operator is applied on two selected individuals, called parents. The selection method used is known as roulette wheel. With this selection technique, the probability for any individual to be selected is directly proportional to its fitness. Once two individuals are selected, they are penalized in order to have less probability of being selected again.

Five types of crossover operators that are applied in a randomly way have been developed. These operators can be described as follows.

Simple crossover. This simple operator interchanges parts of the parents to create the children. As the length of the individuals does not have to be equal, breaking points, inside the range of the smallest individual, have to be chosen, as can be seen in Fig. 3.

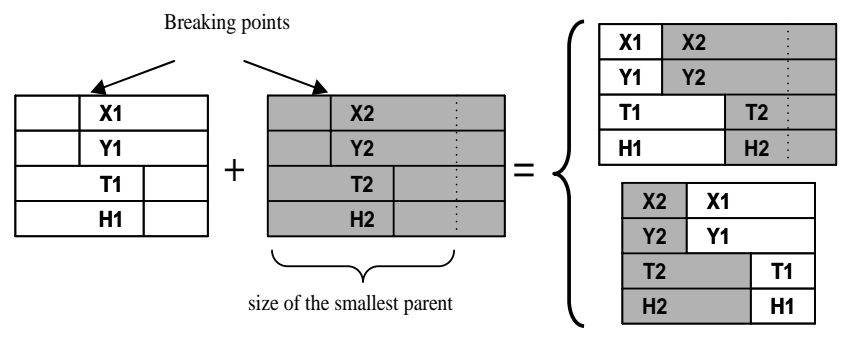

Fig. 3. Simple crossover.

Average crossover. In this crossover operator, the parents are arithmetical averaged between them, so their chromosomes are not interchanged. In this case, it is not necessary to choose any breaking point.

If the parents are different sized, then the children are averaged between the smallest parents with a part of the biggest. So, the size of the son will be the minor size of the parents, as can be seen in Fig. 4.

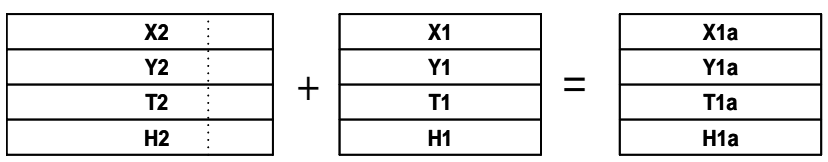

Fig. 4 Average crossover.
Union crossover. This operator joins the parents, so that the size of the son is equal to the sum of the parents' size. So, the union is a concatenation of the parents, as can be seen in Fig. 5.



Fig. 5. Union crossover.

Mixed union crossover. This operator is similar to the simple crossover. However, the breaking points are different for every parent. The difference is that the sizes of the sons are different from the parents, as can be seen in Fig. 6.

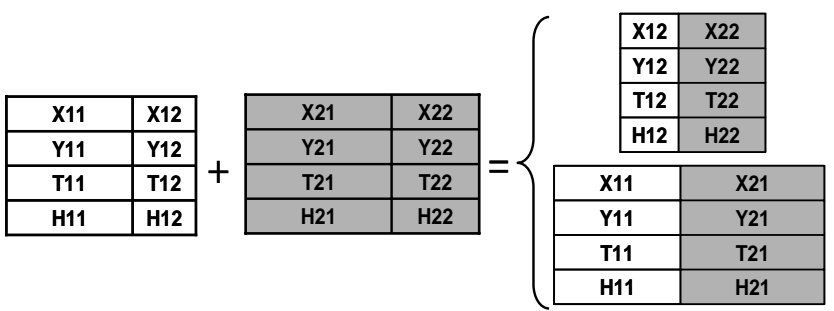

Fig. 6. Mixed union crossover.

\section{Interchange crossover.}

The last crossing operator interchanges elements of the parents, applying a random mask. In this case, multiple breaking points are chosen. The mask is a matrix of zeros and ones, where ones indicate the elements to be interchanged and the size corresponds to the smallest parent one (Fig. 7).

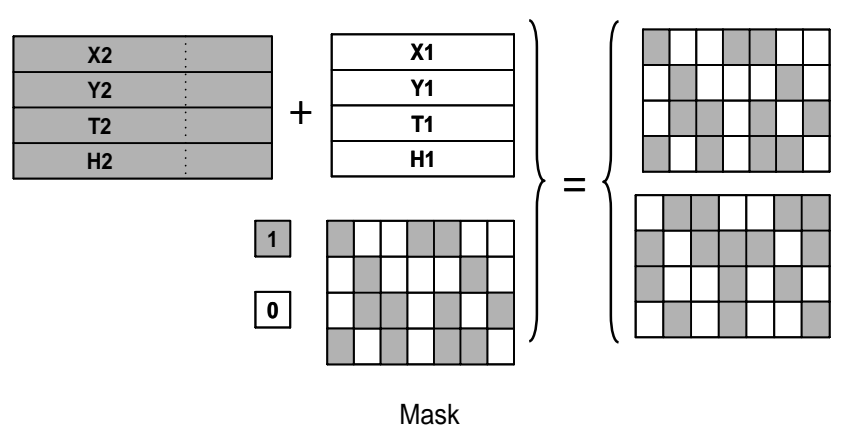

Fig. 7. Interchange crossover.

\section{Mutation operator}

Since during the evolution (from the initial population to the optimum solution) accomplished by the algorithm must lead the natural selection that crosses individuals over the randomness introduced by the mutation, the probability that the mutation operator acts on an individual must be small. Nevertheless, the mutation is an important instrument, because allows to create 
individuals different from the previous population. When the population is confined in a local maximum, this operator leads to the creation of individuals out of this zone of local atraction. This way the EA evolves towards the global maximum.

When a mutation operator acts on an individual, some of it characteristics are modified. A mask selects the properties that are going to change, like in the interchange crossover. Every property (position, type of machine or height) can change around its original value randomly, but in a limited way (Fig. 8).

As expected, after the operations of mutation or crossover, not valid solutions can be created. For example, a wind generator could be place out of the zone of study, or the tower height is incompatible with the type of machine, or several wind generators could be place at the same position, etc. In all these cases, a regenerative algorithm is applied turning the unfeasible individual into a feasible one.

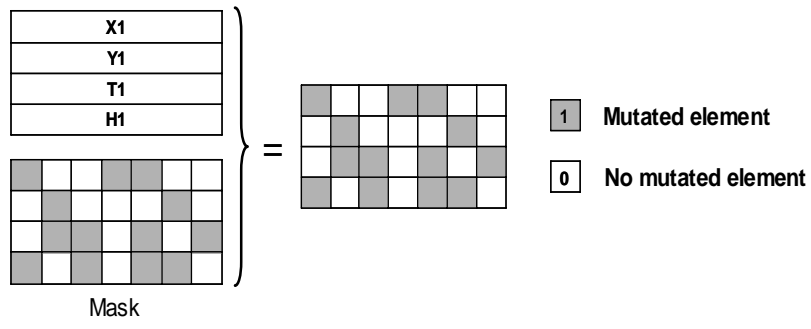

Fig. 8. Mutation operator

Finally, when the best individual does not change in a number of generations, the EA is stopped.

\section{A hybrid evolutive algorithm for wind farm optimum network design}

Taking as a starting point the previously existing high voltage lines and the location of the wind generation points, the algorithm must try to find, looking for the minimum cost, the optimum number and location of the substations, the best layout of the high-voltage lines between the substations and the existing lines, and the best layout of the low-voltage lines between the substations and the individual wind generation points. At the same time, the solution layouts must fulfil all the restrictions about prohibited areas affected with legal, safety, ecology or building limitations.

The total cost considers three main terms, namely highvoltage network cost, substation cost and low-voltage grid cost:

Total Cost $=H V$ Cost $+S B$ Cost $+L V$ Cost

The high and low voltage lines' costs consider:

- The connection costs at the beginning and at the end of the line $\left(C_{A}, C_{B}\right)$. They are present in each section and their value depends on the line voltage.
- $\quad$ The installation costs $\left(C_{H V f}, C_{L V f}\right)$. They consider the material costs and the human resources costs. They mainly depend on the line length.

- The variable costs $\left(C_{H V v}, C_{L V v}\right)$. They consider the exploitation costs and mainly depend on the line length and the power flow.

So, high and low voltage lines' costs can be expressed as:

$$
\begin{aligned}
& H V \text { Cost }=\sum_{H V \text { Lines }}\left(C_{A}+\left(C_{H V f}+C_{H V V} P_{l}\right) \text { Length }_{l}\right) \\
& L V \text { Cost }=\sum_{L V \text { Lines }}\left(C_{B}+\left(C_{L V f}+C_{L V V} P_{l}\right) \text { Length }_{i}\right)
\end{aligned}
$$

The substation costs consider:

- The building costs for the transformer substations $\left(C_{C O}\right)$. These costs are constants; however they are part of the optimisation problem because the number of substations is unknown.

- The transformer LV bus costs $\left(C_{S B}\right)$. Each lowvoltage line is connected with a transformer LV bus.

So, the substation cost can be expressed as:

$$
S B \text { Cost }=\sum_{s b}\left(\text { Bus }_{i} C_{S B}+C_{C O}\right)
$$

In order to solve this coupled problem, a hybrid method is proposed. This method calculates the location of the substations and the low voltage distribution sequentially. An evolutive algorithm calculates the low-voltage distribution grid and works with a numeric method that places the substations.

\section{A. Evolutive Algorithm}

A previously mentioned, genetic algorithms are robust algorithms that find the minimum or the maximum of a function [5,6]. These algorithms use multiple directions of search instead of the only one direction, and use to work with a codified structure of the variables instead of his real values.

\section{1) Codification}

An evolutionary algorithm is used to find the optimum layout of the low voltage lines. It uses the following codification: each every possible solution of the problem is represented by means of a vector, $A_{i}$, as shown in Fig. 9. The size of $A_{i}$ is $2 n p$ (twice the generation points). The first $n p$ elements are a permutation of the $n p$ wind generation points and the second $n p$ elements codify the connection points. The $a_{i j}$ and $a_{i j+n p}$ elements of $A_{i}$ are related and they represent the wind generators and its connection, respectively. When one of the second $n p$ elements is a positive number $\left(a_{i j+n p}>0\right)$ means that the wind generator $\left(a_{i j}\right)$ is connected to a substation. To indicate that the connection is to another point, a negative $\left(a_{i j+n p}<0\right)$ number is used.

To maintain the radial structure of the network, the connections between points are limited: each point only can be connected with a previous point of its 
permutation. For example, for example $a_{i j+n p}=-4$ means that the point $j$ of the permutation is connected with the fourth point of this permutation. $a_{i j+n p}=4$ means that the point $j$ of the permutation is connected with the substation 4 . These conditions limit the values of $a_{i j+n p}$ to the array $:\{-j+1,-j+2, \ldots,-1,1,2, \ldots, n s\}$, when $n s$ is the number of substations. An example of this codification is shown in Fig. 9.

This codification system always generates a radial structure in the network, avoiding spending time in a later checking process. Besides, this codification does not consider neither the coordinates of the substations nor their connections to the high-voltage lines. The criterion is to have, at most, the same number of substation as existing lines, and the substation $k$ is connected to the $k$ line. This codification is enough to run the genetic algorithm to calculate the exact coordinates of the substations, as will be shown later.



Parent $\mathrm{i}=\left[\begin{array}{llllll}2 & 1 & 3 & 1 & 1 & -2\end{array}\right]$

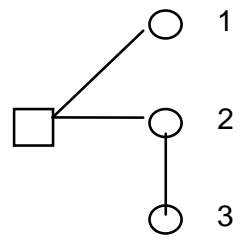

Children $_{i}=\left[\begin{array}{llllll}2 & 1 & 3 & 1 & 1 & -1\end{array}\right]$

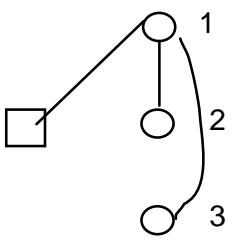



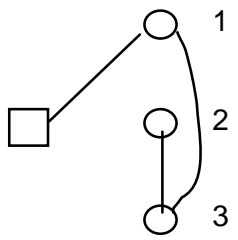

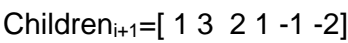

Fig. 9. Individual codification and crossover.

\section{2) Selection and crossing operators}

The crossover operator is applied on two selected individuals, namely parents. Genetic algorithms are basically a mechanism of search based on the species evolution. This evolution is based on selecting the individuals whose characteristics will be present in the next generation.

The choice of the function that relates the cost of a solution with the probability of this selection is not trivial. In this paper the individuals are ordered by their goodness and each individual is crossed with the following individual. In this way, once the percentage of individuals to eliminate in a generation is fixed (tax of elimination), it is possible to know the individuals to cross.

The crossover process is simple: a randomly generated number between the number of wind generators, $n p$, and $2 n p-1$ is selected. This number is used as the start position to begin the interchange of digits inside the array of numbers that represent the solution. When the position is bigger than the number of wind generators the interchange will be out of the permutation area.

This crossover procedure generates two "children" solutions. The first one is a copy of the first elements (up to the crossing position) of the first "parent" solution. The rest of elements are the last elements (from the crossing position to the end) of the second "parent" solution. The elements of the second "child" solution are the elements discarded in the first one.

One example of this crossover procedure is showed in Fig. 9, where the randomly selected position is number 5 . It is possible to see than the children have characteristics from the parents and one's own thanks to the crossing. Different rates of crossing, between $50 \%$ and $100 \%$, have been used in the proposed algorithm, and the best results have been obtained with a tax next to $75 \%$.

\section{3) Mutation operator}

Once the crossovers have been done, the resulting solutions are mutated. Two random numbers are used to perform the mutations. The first set the individual to be mutated, while the second set the individual's gene to be mutated. It is interesting to point that one of the copies of the best individual is not allowed to mutate. This is done in order to keep the best solution.

The mutation operation is performed depending on the gene's position:

a) If the gene belongs to the ones that set the generation point (i.e., subjected to permutation), other random number lesser than $n p$ is generated and the mutation operation interchange the points placed at those positions. As an example, in Fig. 10 the gene to be mutated is number 3 , so a random number, 1 , must be generated.

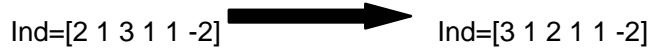

Fig. 10. Mutation operator.

b) If the gene belongs to the group that set the connections of the wind generators, a guided mutation is performed. This way, certain favourable branches are given a higher probability. To do that, each possible mutation fitness is computed in an approximate way.

The probability of the mutation operator acting on an individual is small as during the evolutive algorithm must dominate the natural selection (that crosses individuals) over the randomness (created by mutation). Nevertheless, the mutation is important since it allows create different individuals from the previous population. When the population is confined in a local maximum, this operator leads to the creation of individuals out of this local maximum. This way the evolutive algorithm evolves towards the global maximum. Finally, when the best individual does not change in a number of generations, the evolutive algorithm is stopped. 


\section{4) Repetition operator}

Due to the nature of the problem, branches can only be included from the farthest points to the nearest points. In consequence, the solution is very influenced by the codification of wind generators' position (the three first digits in the individual codification, Fig. 9-10). To avoid that situation, the best individual is copied changing the codification form. In the first solution, the points are ordered depending on the distance form the point to the nearest substation, starting from the first substation and finishing by the last. In the second solution, the points are ordered in the same way, but this time inverting the previous order. This way, each point has the same probability to change of substation.

\section{B. Substations placement}

To evaluate the cost associated to each solution it is required to set the optimal substations placement from the information generated by the genetic algorithm. The coordinates of the substation $k,\left(x_{0 k}, y_{0 k}\right)$, are chosen in order to minimize the following cost,

$C_{H V v}, C_{H V f}$ : Fixed and variable costs related to $\mathrm{HV}$

$C_{L V V}, C_{L V f}$ : Fixed and variable costs related to $\mathrm{LV}$

$P_{k}$ : Power transformed by the substation

$P_{j}$ : Power injected at node $j$

$$
\begin{aligned}
& \left|a_{k} x_{0 k}+b_{k} y_{0 k}-c_{k}\right| \text { : Distance from the } \\
& \text { substation coordinates } \\
& \left(x_{0 k}, y_{0 k}\right) \text {, to the } \mathrm{HV} \text { line } \\
& \left(a_{k} x+b_{k} y=c_{k}\right) \text {. } \\
& L_{j}=\sqrt{\left(x_{0 k}-x_{j}\right)^{2}+\left(y_{0 k}-y_{j}\right)^{2}} \quad \begin{array}{l}
\text { Distance from } \\
\text { substation to wind }
\end{array} \\
& \text { generator/node } j \text {. }
\end{aligned}
$$

As can be seen, the whole LV route is not needed. Only the generators connected to the substation and its power are needed. It must be observed that the function cost includes the fixed and variable cost related to the HV line (from the HV line to the substation) and the cost related to the LV line (form substations to wind generators). The costs of every transformer substation bus are included in the main algorithm. The fixed costs related to the beginning and end of the $\mathrm{HV}$ and $\mathrm{LV}$ lines are not considered because they are constant and do not determine the substation placement.

The process to compute the coordinates $x_{0 k}$ and $y_{0 k}$, is not especially difficult but is very time consuming, especially when it must bee repeated a high number of times. So, this coordinates are only computed in the last generation of the genetic algorithm. Previously, an approximated value is considered. This approximation is based on the electric gravity centre, $\left(x_{g c}, y_{g c}\right)$, defined as:

$$
x_{g c}=\sum_{i} w_{i} x_{i} \quad y_{g c}=\sum_{i} w_{i} y_{i} w_{i}=\frac{\left(P_{i} C_{L V v}+C_{L V f}\right)}{\sum_{i}\left(P_{i} C_{L V V}+C_{L V f}\right)}
$$

$P_{i}$ : Power injected at node $i$

$C_{L V f}: \mathrm{LV}$ fixed costs related to the conductor path and poles

$C_{L V V}: \mathrm{LV}$ variable costs related to line power losses
Taking into account the electric gravity centre, three possible substation placements are considered:

a) The intersection point between the $\mathrm{HV}$ line and the perpendicular from the gravity centre, $\left(x_{L}, y_{L}\right)$, as shown in Fig. 11a.

b) The same gravity centre coordinate, $\left(x_{g c}, y_{g c}\right)$, as in Fig. 11.b.

c) A point between both, $\left(x_{I}, y_{I}\right)$, as in Fig. 11c. The position is calculated as a result of the following weighting between the $\mathrm{HV}$ and the $\mathrm{LV}$ costs.

$$
\begin{aligned}
& x_{I}=x_{L} \lambda+x_{g c}(1-\lambda) \quad y_{I}=y_{L} \lambda+y_{g c}(1-\lambda) \\
& \lambda=\frac{C_{H V V} P_{i}+C_{H V f}}{\left(C_{H V V}+C_{L V V}\right) P_{\mathrm{i}}+C_{H V f}+C_{L V f} N b}
\end{aligned}
$$

$P_{i}$ : Power injected at node $i$

$\mathrm{Nb}$ : Number of branches

$C_{L V f}$ : LV fixed costs related to the conductor path and poles

$C_{L V v}: \mathrm{LV}$ variable costs related to line power losses

$C_{H V f}: \mathrm{HV}$ fixed costs related to the conductor path and poles

$C_{H V V}$ : HV variable costs related to line power losses

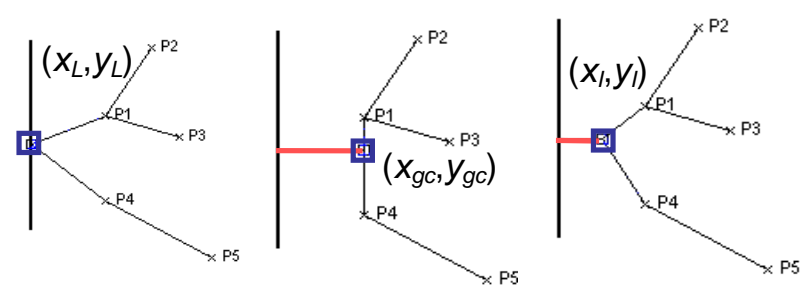

Fig. 11. Substation placement.

The placement with the minimum value of $Z_{k}$ is chosen. This computation is repeated for each active substation. When a main line is overloaded, exceeding its maximum transmission power flow, a penalty is added to the total cost.

\section{Forbidden zones}

The optimum configuration of the wind power distribution network could be limited due to the setting of certain "forbidden zones" were the presence of the grid is not allowed (i.e., physical obstacles in the path, presence of buildings or safety and other regulations). The treatment will be different depending on the voltage level.

- A HV branch crossing through a forbidden zone. The connection between the origins to the target points is redesigned getting around the forbidden zone.

- A LV branch crossing through a forbidden zone. The genetic algorithm set the probability of any branch that crosses a forbidden zone to null (in the initial solution generation and the mutations). This does not avoid the possibility that, after a crossing operation, one of those forbidden branches appears. So, the evaluation of the solutions must test the presence of this kind of violations. When the presence of one of these forbidden branches is detected, its cost is 
doubled. Other constrains such as thermal limits and voltage drops are considered by penalties.

\section{Test case}

The case selected is a plane area with a main road as is shown in the Fig. 12 (red line). The cost considered for the main road are $50.000 €$ and a coefficient of $700 € / \mathrm{m}$ for secondary roads. The distribution of the wind is as shown in Fig. 13.

The shape parameter has been considered the same for all locations $(K=5)$.

Figure 14 shows a Weibull approximation of the wind speed histogram (frequency or percentage of time), measured at $50 \mathrm{~m}$ height. The scale and shape parameter are $C=14 \mathrm{~m} / \mathrm{s}$ and $K=5$, respectively.

The features of the considered wind turbine are shown in the Table I, including the wind speed-power characteristic.



Fig. 12. Land selected for establisment of the Wind Farm.

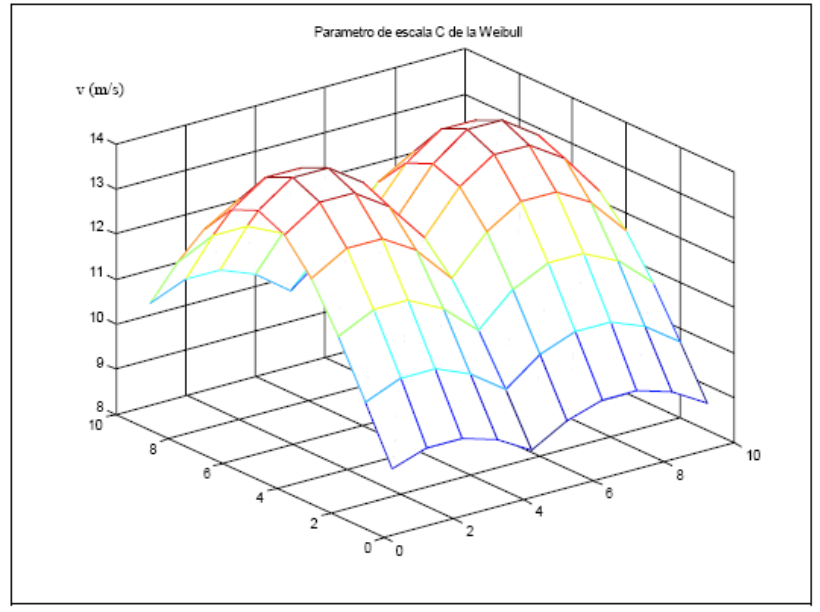

Fig. 13. Distribution of the wind

Figure 15 shows the best solution found and the cost of the Wind Farm without taken into account the cost of the conection network.

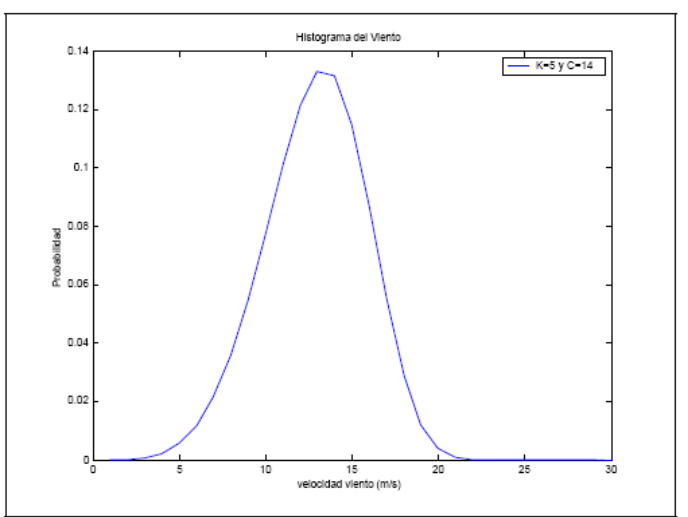

Fig. 14. Weibull approximation of the wind speed histogram.

Table I. Features of the machine considered.

\begin{tabular}{|l|l}
\hline \multicolumn{2}{|c}{ Datos de máquinas en maquinas.dat } \\
\hline Marca & NEG-Micon \\
\hline Modelo & NM 600 (Paso Fijo) \\
\hline Potencia (kW) & 600 \\
\hline Altura minima & 30 \\
\hline Altura máxima & 100 \\
\hline Coste (euros) & 400000 \\
\hline Coste torre (euros/m) & 1000 \\
\hline Coste cimentación (euros) & 70000 \\
\hline Curva caracteristica de potencia & 00021.249 .383 .2130 .7202 .0280 .8361 .6 \\
& 433.7498 .6548 .1577 .3596 .0602 .0601 .9 \\
593.4571 .3545 .6524 .7510 .0500 .7478 .7 \\
457.7
\end{tabular}

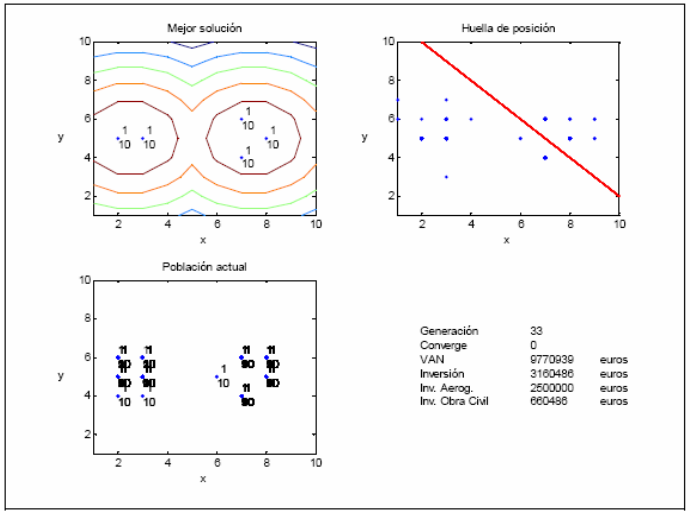

Fig. 15. Best solution for the wind turbine sitting problem.

For the optimum network design, two HV evacuation lines are considered as is shown in the Fig. 16.

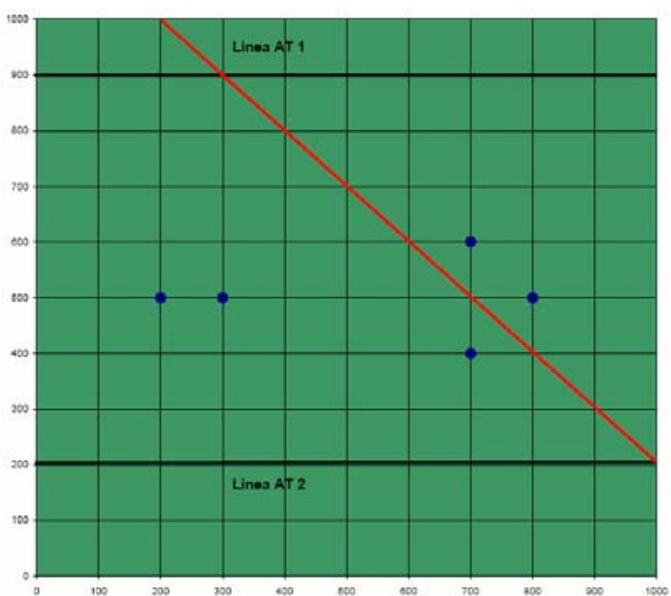

Fig. 16. Generator points, main road and existing HV lines. 
For this optimum case, the considered costs are:

- The connection costs at the beginning and at the end of the line $\left(C_{A}, C_{B}\right): C_{A}=6000 € ; C_{B}=6000 €$.

- The installation costs $\left(C_{H V f}, C_{L V f}\right): C_{H V f}=60000 €$; $C_{L V f}=6000 €$.

- $\quad$ The variable costs $\left(C_{H V v}, C_{L V v}\right): C_{H V v}=6000 € / \mathrm{km}$; $C_{L V V}=9000 € / \mathrm{km}$.

- The substation costs: $C_{S B}=240000 €$.

Table II. Distribution of the network cost.

\begin{tabular}{|c|c|c|c|c|c|c|c|}
\hline Section & Start & End & $\begin{array}{c}\text { Power } \\
(\mathbf{k W})\end{array}$ & $\begin{array}{c}\text { Length } \\
(\mathbf{m})\end{array}$ & $\begin{array}{c}\text { Total } \\
\text { Cost } \\
(\boldsymbol{(})\end{array}$ & $\begin{array}{c}\text { Variable } \\
\text { Cost } \\
(\boldsymbol{(})\end{array}$ & $\begin{array}{c}\text { Fixed } \\
\text { Coste } \\
(\boldsymbol{(})\end{array}$ \\
\hline 1 & 1 & 2 & 600 & 100 & 7140 & 540 & 6600 \\
\hline 2 & 2 & 3 & 1200 & 410 & 12930 & 4450 & 8470 \\
\hline 3 & 3 & Sb1 & 3000 & 200 & 12600 & 5400 & 7200 \\
\hline 4 & 4 & 3 & 600 & 200 & 8280 & 1080 & 7200 \\
\hline 5 & 5 & 3 & 600 & 140 & 7610 & 760 & 6850 \\
\hline 6 & LAT 2 & Sb1 & - & 0 & 6000 & 0 & 6000 \\
\hline
\end{tabular}

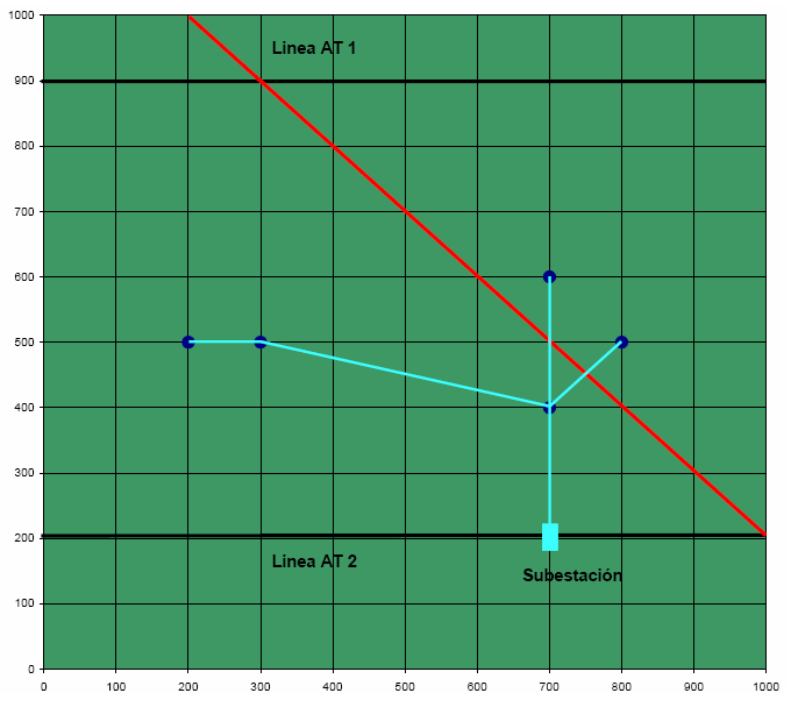

Fig. 17. Optimum wind farm network design.

Figure 17 shows the optimum network configuration found and Table II shows the cost components.

\section{Conclusion}

Although wind farm design is a well known problem its solution used to be heuristic, based on the designer experience. In this work the global optimization wind farm design problem has been splitted in two main (uncoupled) sub-problems. The first steep is the optimum wind turbine sitting and the second, the optimum wind farm network configuration.

The first problem, selection and placement of the wind turbines, has been solved with an evolutive algorithm. This algorithm can set the layout of a wind farm with minimum investment and most efficient use of the wind resource. To reach this goal, the algorithm should be able of choosing, among the available wind turbines, the one that offer better performance with a smaller price for the available data wind. Three main cost components have been taken into account for every wind turbine. First, a fixed cost associated to the own machine. A second component related to the variable tower height (meters), and finally, the installation component, depending on the generator location.

The second problem, the wind farm optimum network design, has been solved with a hybrid numerical evolutive algorithm method. The numerical method designs the HV installation, while the $\mathrm{LV}$ is calculated with an evolutive algorithm.

The proposed algorithms consider the fixed and variable costs associated to the solution, along with the problem constrains.

As a result, a global two-steeped method has been developed which is able to profit the wind farm investment in an optimum form.

\section{Acknowledgement}

The authors would like to acknowledge the financial support provided by Spanish MCYT and Junta de Andalucía, under grants ENE2004-03342/CON, DPI2002-04416-C04-02 and ACC-1021-TIC-2002.

\section{References}

[1] N. Jenkins, "Electrical Design of Wind Farms", Proc. IEEE/NTUA Athens Power Tech Conference, Athens, Greece, pp. 990-994, Sep. 5-8, 1993.

[2] N. Jenkins, "Engineering Wind Farms", Power Engineering Journal, pp. 53-60, April, 1993.

[3] J. Castro Mora, J.M. Calero Barón, J.M. Riquelme Santos, M. Burgos Payán. "An evolutive algorithm for wind farm optimal design". Special Issue on Electrical Engineering of Neurocomputing NEUCOM-D-05-00328R2, Elsevier.

[4] El-Khattam, W.; Bhattacharya, K.; Hegazy, Y.; Salama, M.M.A. "Optimal investment planning for distributed generation in a competitive electricity market", IEEE Transactions on Power Systems, Volume 19, Issue 3, Aug. 2004, pp. $1674-1684$.

[5] El-Khattam, W.; Hegazy, Y.G.; Salama, M.M.A. "An integrated distributed generation optimization model for distribution system planning", IEEE Transactions on Power Systems, Volume 20, Issue 2, May 2005, pp. 1158-1165.

[6] Ponnavaikko, M., Prakasa Rao, K.S., and Venkata, S.S.: "Distribution system planning through a quadratic mixed integer programming approach", IEEE Trans. Power Delivery, 1987, 2, (4), pp. 1157-1163.

[7] Thompson, G.L., and Wall, D.L.: "A branch and bound model for choosing optimal substation locations", IEEE Trans. Power Apparatus and Systems, 1981, 100, (5), pp. 2683-2688.

[8] S. Heier, "Grid integration of wind energy conversion systems", John Wiley and Sons, 1998.

[9] D.E. Goldberg, "Genetic algorithms in search, optimization and learning", Addisson-Wesley Pub. Co. Inc, 1989.

[10] R. Spillman, "Genetic algorithms, nature's way to search for the best". Dr. Dobb's J 1993:26-30

[11] J.J. Grefenstette, "Optimization of Control Parameters for Genetic Algorithms", IEEE Trans. On Systems Man, And Cybernetics, vol. SMC-16, pp. 122-128, Jan./Feb. 1986. 